\title{
Technical note: Creating a four-dimensional model of the liver using finite element analysis
}

\author{
K. K. Brock ${ }^{\text {a) }}$ \\ Department of Radiation Oncology, University of Michigan, Ann Arbor, Michigan 48109 \\ S. J. Hollister \\ Department of Biomedical Engineering, University of Michigan, Ann Arbor, Michigan 48109 \\ L. A. Dawson and J. M. Balter \\ Department of Radiation Oncology, University of Michigan, Ann Arbor, Michigan 48109
}

(Received 31 October 2001; accepted for publication 18 April 2002; published 20 June 2002)

\begin{abstract}
Finite element analysis and two liver CT scans were used to construct a four-dimensional (4D) model of the liver during breathing. A linear elastic, small deformation mechanical model was applied to one patient to obtain intermediate organ position and shape between exhale and inhale. Known transformations between anatomically defined subsections of the exhale and inhale liver surfaces were applied as constraints to the exhale CT liver model. Intermediate states were then calculated and time weighted to determine a 4D model of the liver as it deforms during the breathing cycle. This model can be used to calculate a more accurate dose distribution during radiotherapy. (C) 2002 American Association of Physicists in Medicine.
\end{abstract}

[DOI: $10.1118 / 1.1485055]$

Key words: organ deformation, organ motion, mechanical modeling

The potential for tumor tracking as well as ventilatory gating of radiotherapy requires an improved understanding of dose deposition in organs that move and deform due to breathing. Both of these types of systems operate with the use of tolerance windows, permitting some residual movement of organs during irradiation. ${ }^{1,2}$ The magnitude of permitted movement is inversely proportional to the length of treatment time, and also impacts the accuracy of modeled dose. Furthermore, it may be possible to optimize radiotherapy plans so that they are less sensitive to breathinginduced geometric variations. Toward these ends, it is useful to generate models of organs describing realistic changes in position and shape, and which can be used for dose calculations using radiotherapy treatment planning systems. Finite element modeling and analysis tools, in routine use for engineering applications, may serve as a mechanism for providing these models. ${ }^{3}$ This example case demonstrates the development of a four-dimensional (4D) model of the liver that undergoes temporal changes in organ shape as well as position, and could potentially be integrated with dose calculation for radiotherapy treatment planning.

Two CT scans were acquired from the same patient under active breathing control. ${ }^{4}$ One scan was at end-exhale and one was at end-inhale. Six regions of the liver were used to determine an initial estimate of the deformation between inhale and exhale. Four of these regions labeled segments II, V, IVB, and VIII were defined from a standard anatomic reference system. ${ }^{5}$ Two additional regions were used to define the posterior medial and superior aspects of the liver as these regions were observed to undergo substantial relative displacements. The magnitude of displacements at these regions were determined using a three-dimensional treatment plan- ning system (UMPLAN, University of Michigan, Ann Arbor, MI).

The liver was assumed to undergo linear, elastic, small deformations only. The assumed isotropic elastic model requires two material properties, the elastic modulus and Poisson's ratio. The elastic modulus of a material represents the relative stiffness of the material within the elastic range and can be determined in a lab setting by calculating the ratio of stress to strain. Poisson's ratio is lateral contraction per unit breadth divided by the longitudinal extension per unit length. The value for the elastic modulus was obtained from studies done by Kruse et al. ${ }^{6}$ The elastic modulus was calculated from the median shear stiffness, $3 \mathrm{kPa}$, obtained at a frequency of $100 \mathrm{~Hz}$. The elastic modulus $(E)$ is related to the shear modulus $(G)$ by the relationship $E=G^{*} 2 *(1+\nu)$. The elastic modulus was assigned a value of $7.8 \mathrm{kPa}$. Poisson's ratio was assigned a value of 0.3 , to allow for a small amount of compression.

The exhale CT data were first converted into stereolithography data that represent surfaces using triangular facets. The surface facet data were then imported into a finite element modeling software package (Hypermesh, Altair Computing, Inc, Troy, MI). A tetrahedral mesh with ten node connectivity was created that spanned the space encompassed by the liver surface. Material properties were then assigned to all elements in the mesh. Regions of the mesh were selected corresponding to the six regions of the liver described previously. Each region was then initially assigned a constraint equal to the transformation of that region, calculated from the difference in position between exhale and inhale. This constraint was then used as a starting point to determine the optimal transformation to create a model of the inhale position that most closely matched the actual patient inhale po- 


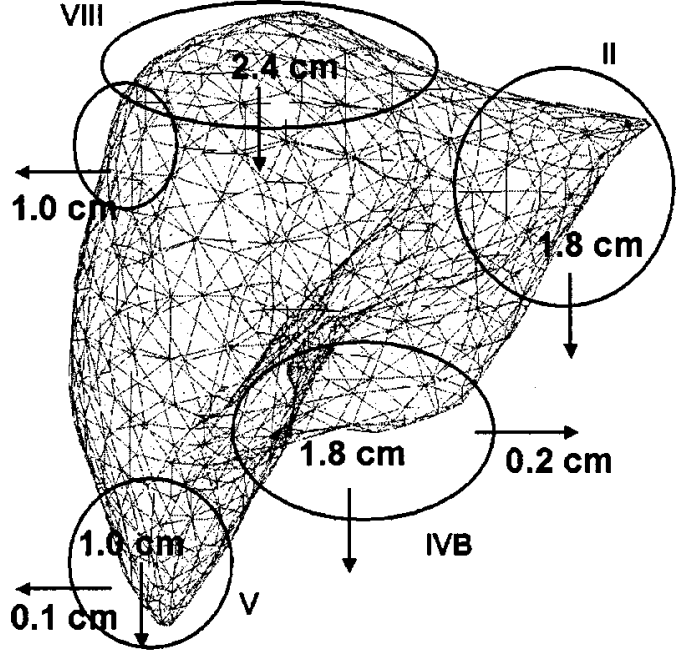

(a)

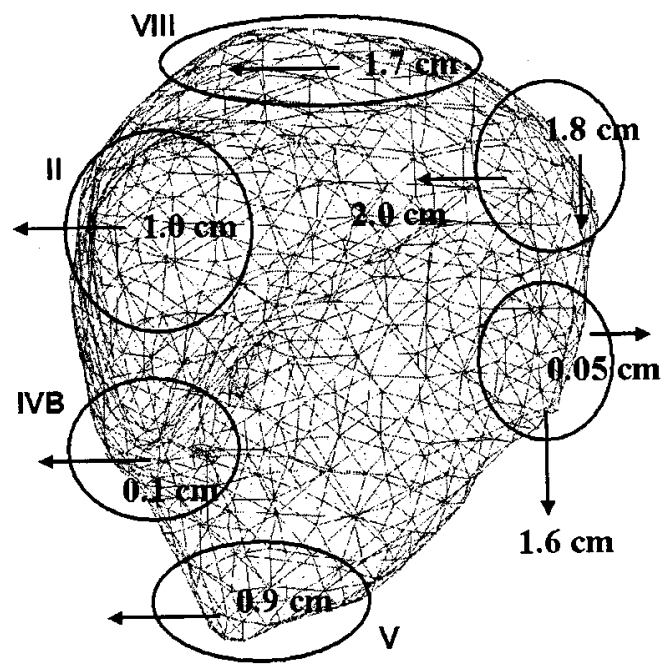

(b)

FIG. 1. (a) AP view of the liver at end-exhale with regions and displacements. (b) Lateral view of the liver at end-exhale with regions and displacements.

sition. After several iterations, final transformations were determined for the six regions [Figs. 1(a) and 1(b)]. Finite element analysis (FEA) software (Abaqus, Hibbitt, Karlsson, and Sorensen, Inc, Pawtucket, RI) was used to determine the displacements, stresses, strains, and reactive forces at each node in the mesh, essentially completing the modeling of displacement of foci that were not directly measured for input.

The modeled inhale liver created from FEA and the actual inhale liver from the CT data were qualitatively compared (Fig. 2). Both the shape and position were evaluated to ensure sufficient information was provided for the transformation. A displacement map was constructed to display the transformation of all liver points, including those not predetermined by the constraints (Fig. 3).

Intermediate states of the liver were then constructed using the finite element modeling software. Each node was moved fractionally along its predicted path from exhale to

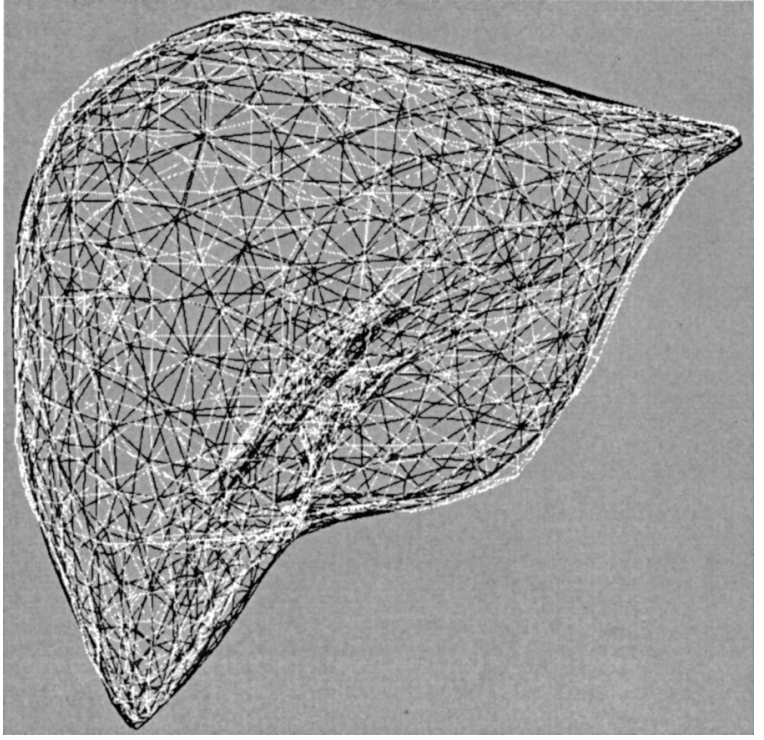

FIG. 2. Modeled inhale position shown in white, actual patient liver at inhale shown in black.

inhale to generate its intermediate position. Six intermediate states are shown here (Fig. 4). A 4D model of the liver was determined by weighting the intermediate states of the liver according to measured free-breathing time courses. ${ }^{7}$ As the liver deforms from the exhale state to the inhale state, the top of the liver moves caudally toward the bottom of the liver. The top of the liver, specifically region VIII, flattens as the liver moves from exhale to inhale. This is due to the contributions of the diaphragm that contracts during inhale and acts as a driving force and the apparent elasticity of the liver in this patient. The liver also increases in medial-lateral width as the top of the liver moves in the cranial-caudal direction. The volume of the liver was reduced by $0.93 \%$ from exhale to inhale.

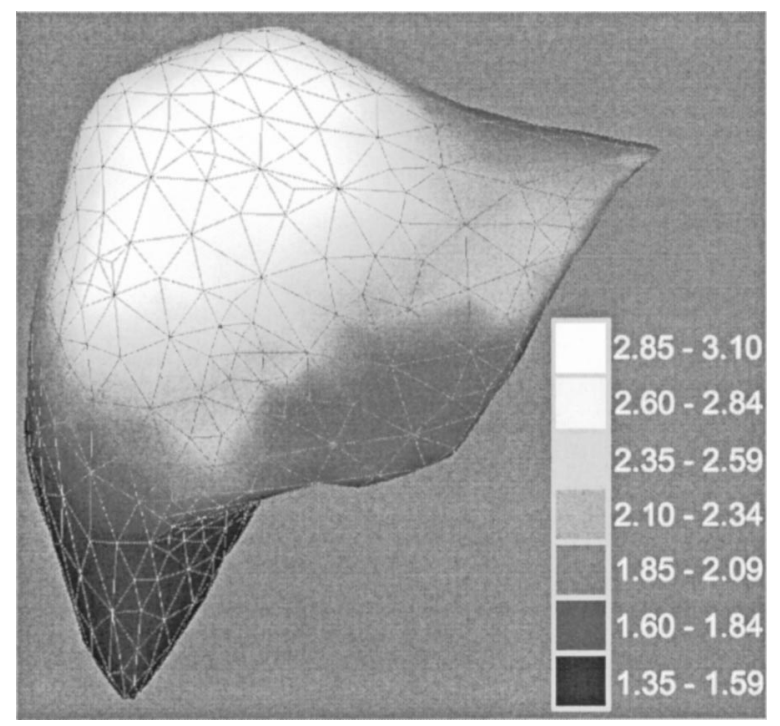

FIG. 3. Displacement map of end exhale liver to modeled end inhale position. Displacements are vector magnitudes and are reported in centimeters. 

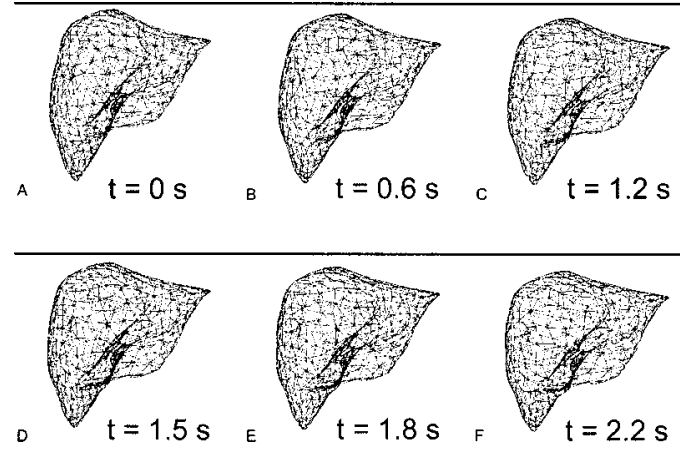

FIG. 4. Six intermediate states of the liver from end exhale (A) to end inhale (F)

Note that the linear elastic model applied assumes a linear path of travel for each node, and that no viscoelastic or nonlinear (material or deformation) behavior was assumed to occur under forces exerted during normal breathing. It will be a goal of future studies to determine whether nonlinear factors significantly influence these models. The model also assumes homogeneous liver material properties. It is expected that liver properties will vary both within a single patient's liver as well as between patients. It will be a goal of future work to determine and incorporate these variations into the patient model.

This method shows that it is feasible to develop a dynamic model of the liver as it deforms from inhale to exhale. Using finite element analysis, intermediate positions of the liver can be calculated if the end-exhale and end-inhale positions are known. Knowing the intermediate states that occur during breathing could better determine how physiological movements affect the dose received by the tumor and the adjacent normal organs during radiation therapy. It is also possible that the bounded states of inhale and exhale may be used to solve for the elastic modulus of subsections of the liver, thus permitting more accurate modeling of individual patients who may have varying disease (and elasticity) of the liver (e.g., cirrhotic livers). Using standard engineering tools such as FEA software not only allows for rapid generation of deformable models, it also permits investigation of more complex biomechanical behavior, such as viscoelasticity. ${ }^{7,8}$

Work supported by NIH Grant No. P01-CA59827003.

${ }^{\text {a)} E l e c t r o n i c ~ m a i l: ~ k k b r o c k @ e n g i n . u m i c h . e d u ~}$

${ }^{1}$ S. S. Vedam, P. J. Keall, V. R. Kini, and R. Mohan, "Determining parameters for respiration-gated radiotherapy," Med. Phys. 28, 2139-2146 (2001).

${ }^{2}$ S. Shimizu, H. Shirato, S. Ogura, H. Akita-Dosaka, K. Kitamura, T. Nishioka, K. Kagei, M. Nishimura, and K. Miyasaka, "Detection of lung tumor movement in real-time tumor-tracking radiotherapy," Int. J. Radiat. Oncol., Biol., Phys. 51, 304-310 (2001).

${ }^{3}$ D. Yan and D. Lockman, "Organ/patient geometric variation in external bean radiotherapy and its effects," Med. Phys. 28, 593-602 (2001).

${ }^{4}$ J. M. Balter, K. K. Brock, D. W. Litzenberg, D. L. McShan, T. S. Lawrence, R. K. Ten Haken, C. J. McGinn, K. L. Lam, and L. A. Dawson, "Daily targeting of intrahepatic tumors for radiotherapy," Int. J. Radiat. Oncol., Biol., Phys. 52, 266-271 (2001).

${ }^{5}$ J. S. Thompson, Thompson's Core Textbook of Anatomy, 2nd ed. (Lippincott, Philadephia, PA, 1990).

${ }^{6}$ S. A. Kruse, J. A. Smith, A. J. Lawrence, M. A. Dresner, A. Manduca, J. F. Greenleaf, and R. L. Ehman, "Tissue characterization using magnetic resonance elastography: Preliminary results," Phys. Med. Biol. 45, 1579-1590 (2000).

${ }^{7}$ J. M. Balter, K. L. Lam, C. J. McGinn, T. S. Lawrence, and R. K. Ten Haken, "Improvement of CT-based treatment-planning models of abdominal targets using static exhale imaging," Int. J. Radiat. Oncol., Biol., Phys. 41, 939-943 (1998).

${ }^{8} \mathrm{~K}$. Miller, "Constitutive modeling of abdominal organs," J. Biomech. 33, $367-373$ (2000). 